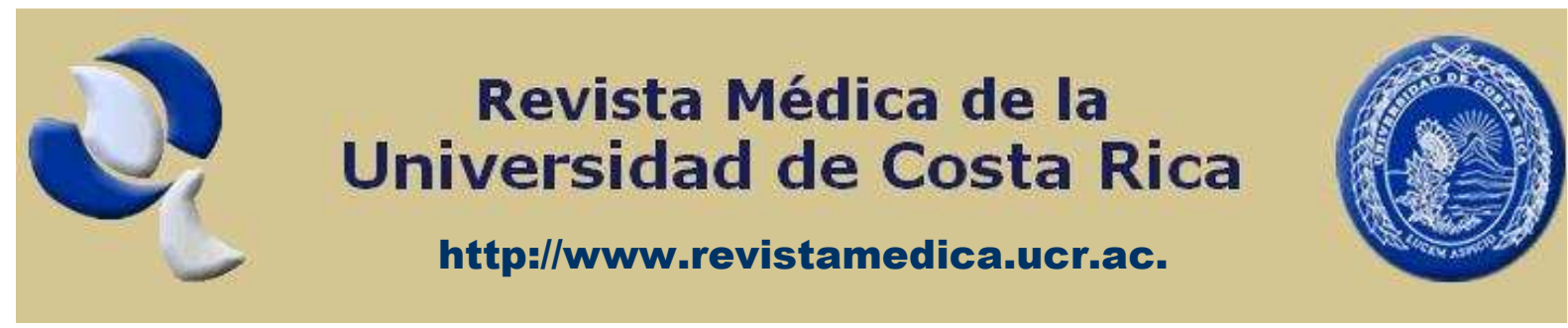

Investigación original

\title{
PERFIL DE PRESENTACIÓN DE LA PENDICITIS AGUDA EN LA PACIENTE EMBARAZADA: Mejorando la exactitud diagnóstica en un Hospital General
}

\author{
Sánchez Arias, Mario \\ Hospital Dr. Rafael Ángel Calderón Guardia. San José, Costa Rica.
}

\section{Resumen}

El diagnóstico exacto y temprano de la apendicitis aguda en la paciente embarazada es difícil, dado que ella tiene una serie de cambios que hace que la respuesta del organismo y la expresión clínica sea menos clara. Una buena historia clínica con un examen físico detallado, repetido en el tiempo por el mismo explorador, más un leucograma con leucocitosis de más de 17.000 y sobre todo la presencia de más de 6 formas en banda, con proteína C reactiva positiva, es lo que nos ayudaría al diagnóstico según esta revisión. Nunca tratar a estas pacientes con analgésico o antibióticos cuando haya la mínima posibilidad de que sea una apendicitis. Cuando no sea suficiente para el diagnóstico, se puede realizar un ultrasonido, sabiendo que si la confirma es bueno, pero si no, no la descarta. En donde este disponible la tomografía axial helicoidal, nos puede aclarar muy bien, si disponemos de un radiólogo experto y un reporte oportuno. Todas las pacientes deberán recibir antibiótico profiláctico que no afecte el producto, en la cirugía producir el menor trauma posible al producto y evitar drenajes o cualquier cuerpo extraño.

Monitorizar muy bien la actividad uterina y toda vez que se desarrollen contracciones, proceder con útero-inhibidores dependiendo de la edad gestacional.

El experto en cirugía laparoscópica puede ingresar por esta vía, con los cuidados descritos para controlar el producto muy de cerca, con la clara idea de convertir ante cualquier riesgo mayor.

reserved. 
Palabras clave: Abdomen agudo, Embarazo, Apendicitis

Recibido: Marzo 2008. Aprobado: Marzo 2008. Publicado: Marzo 2008.

\section{Abstract}

The diagnosis of apendicitis in the pregnant patient is always difficult because the changes that this kind of patient have.

A good clinical history with a very careful physical examination for the same observer plus a white count of 17.000 and more than 6 inmature white cels p.c.. An C protein (+), should be good for making the diagnosis.

Never treat these patients with antibiotics o analgesics before the surgeon evaluation.

When those test are not enough we can send an ultra sound but if it is negative do not means that the diagnosis is wrong.

Some places have CT scan an radiologist who can report inmediatly, if this is the case could be reasonable.

When the surgeon decide surgery we have to give all these patients profilactic antibiotic. In the procedure not disturb the uterus and not leave drainages.

Check carefully the uterus activity after the surgery and give medication if there are contractions.

The expert in laparoscopic surgery can work by this means but had to have the convertion of the procedure as a tool whenever face any risky conditions.

Keywords: acute abdomen, pregnancy appendicitis

\section{Introducción}

Para hacer diagnóstico de alguna patología, lo primero es sospecharla, pensar en ella y esto es de mayor relevancia en la apendicitis aguda durante el embarazo, ya que hay varios cuadros clínicos que pueden confundirse. Tomando en cuenta que la literatura nos dice que los procesos agudos intra-abdominales de causa noobstétrica se ven 1 en 500 embarazos [1], debemos estar alerta a su aparición en esta población. Sobre todo porque como se ha definido desde hace muchos años: la mortalidad de estos procesos en la pacientes embarazada, es la mortalidad del retrazo, $11 \%$ globalmente $[2,3,4,5,6]$. Concretamente refiriéndonos a la apendicitis aguda
(ApAg), los datos publicados muestran un promedio de 1 caso por cada 1.500 embarazadas $[4,5,6,7,8,9,10]$.

Al hablar de ApAg. en la embarazada debemos pensar en toda la fisiopatología de su desarrollo, más los cambios que se dan, por el embarazo $[5,11]$. El abdomen agudo que se produce cuando se desarrolla una ApAg es de tipo peritonítico y raramente puede presentar datos obstructivos. Es de suma importancia distinguir si hay peritonitis, dado que la pérdida fetal es de solo $1.5 \%$ si no la hay y aumenta hasta $30-50 \%$ cuando hay presencia de la misma. $[5,6,8,12,13]$. Pudiéndose decir que el riesgo del feto es

Revista electrónica publicada por el Departamento de Farmacología de la Escuela de Medicina de la Universidad de Costa Rica, 2060 San José, Costa Rica. ® All rights 
exponencial conforme se retrasa el diagnóstico y se resuelve el caso.

La edad promedio de presentación de la ApAg en la mujer embarazada es de 23 años [6], no hay razón fisiopatológica para una mayor presentación de esta patología en alguno de los tres trimestres, pero las distintas series coinciden en que tiene una leve predominancia en el II trimestre $[8,9,10,14,15]$, con un índice de perforación tres veces más frecuente que en la mujer de la misma edad no embarazada. Si bien se acepta un $15 \%$ [16] de diagnósticos equivocados en la mujer joven por la patología tuboovárica, en la mujer grávida ese porcentaje puede ascender hasta 23$30 \%[2,6]$, pues el embarazo agrega un gran factor de error diagnóstico $[4,5,7,9,17]$. La morbilidad materna es mayor en la población embarazada que en la no embarazada ( $9.6 \%$ vrs $0.3 \%$ ) [6], pero la mortalidad materna es muy similar al grupo femenino, dado que esta población constituye un grupo más joven $[16,18]$.

A pesar de que el diagnóstico sigue siendo fundamentalmente clínico, hay variación de la sintomatología y signología con respecto a la mujer no embarazada $[6,17,19]$. El cambio de la posición de la apéndice conforme crece el embarazo produce sintomatología diferente (no necesariamente focalizada en fosa ilíaca derecha) $[5,20]$ y en algunos casos a cuadrante superior derecho, si el embarazo es avanzado o existe una posición retrocecal $[11,21]$. Se acompaña de náusea y vómitos que también son frecuentes en el embarazo en $70 \%$ [22] y febrícula que también se presenta en la mujer grávida. Usualmente en la mujer embarazada se dan alteraciones en el examen general de orina y la leucocitosis que tanta ayuda nos da en la mujer no embarazada, resulta que se presenta en la embarazada hasta cifras de 15.000. Puede ser de ayuda en este particular el predominio de neutrófilos ( $80 \%$ o más) $(5,23)$ y la presencia de bandas y formas inmaduras, las cuáles son sugestivas de cuadro inflamatorio peritoneal, todo lo cuál confunde el diagnóstico.

Con el uso del ultra-sonido compresivo como medio diagnóstico en la mujer embarazada en que se sospecha apendicitis aguda, hemos agregado un factor de ayuda, que en buenas manos puede dar sensibilidad de hasta 78$100 \%$ y especificidad de $89-96 \%$ [24], más no de exclusión, pues es un estudio muy dependiente del operador, del equipo que se use, de la existencia o no de gas y de la presencia de al menos edema de la pared apendicular. De tal suerte que si lo diagnostica, perfecto, pero si no, no lo descarta. Si es muy útil para diferenciar otras patologías que se pueden confundir con apendicitis, especialmente del orden de patología tubo-ovárica. El ultra sonido transvaginal puede tener mejor sensibilidad, pero de nuevo es muy dependiente del operador y su disponibilidad [11].

Mención especial debe hacerse en relación a la posibilidad de usar la tomografía axial computarizada de tipo helicoidal, la cual si bien produce radiación de 300 mrad por estudio, es menor de lo aceptado como riesgo en la mujer embarazada (5-10 rad). Además, si se dispone de un radiólogo con experiencia, puede darnos una exactitud diagnóstica de hasta el $98 \%$, pero con el consecuente mayor costo y retraso en la resolución quirúrgica $[11,25,26]$. La Resonancia Magnética Nuclear, usada con medio de contraste oral, también puede usarse con sensibilidad de 100\% y especificidad $93.6 \%[27,28]$.

Revista electrónica publicada por el Departamento de Farmacología de la Escuela de Medicina de la Universidad de Costa Rica, 2060 San José, Costa Rica. ${ }^{\circledR}$ All rights reserved. 
Con el advenimiento de la cirugía laparoscópica, se ha usado la laparoscopía como una herramienta más de diagnóstico y en grupos con experiencia hasta terapéutica $[29,30]$ con ventajas potenciales de: rápida recuperación, rápida movilización, menos tromboembolismo, menor requerimiento narcótico, corta hospitalización, menos problemas de herida, menos movilización uterina. $Y$ algunas desventajas como pueden ser: toxicidad por el dióxido de carbono, aumento de presión intra-abdominal y disminución de irrigación al producto,

\section{Objetivo}

Evaluar las características clínicas y diagnósticas de la mujer embarazada con apendicitis.

\section{Método y Material}

Se han revisado retrospectivamente todos los pacientes que egresaron del año 2000 al 2006 con el diagnóstico de Apendicitis Aguda y Embarazo de los servicios de cirugía del Hospital Dr.R.A.Calderón Guardia.

Evaluando en todos ellos: edad de presentación, estancia hospitalaria, la clínica presentada, tiempo de evolución

\section{Resultados}

Se encontraron 42 pacientes con ese diagnóstico y una tasa de 6749 partos por año, lo que nos da una incidencia de 6 casos por año, con una relación de 1 caso por cada 1124 partos. Del total de casos solo se pudieron estudiar 27 $(64.2 \%)$. compresión a cava inferior, posible daño directo al útero y al producto [22].

Al igual que en la paciente no embarazada, se debe descartar otras patologías posibles tales como: colecistopatía aguda, enfermedad ulcerosa, reflujo, gastritis, pancreatitis, colitis y otras propias del embarazo que no son quirúrgicas: dolor ligamentoso, contracciones Braxton Hicks $[5,11,13,15,19,31]$ y estar lo más seguro posible del diagnóstico para no dejar de operar, pero tampoco operar a quien no lo necesita, con el fin de no agregar morbilidad $\mathrm{y} / \mathrm{o}$ mortalidad fetal [4\%].

Diseño: Observacional, retrospectivo y descriptivo de registros médicos.

de la enfermedad, antecedentes mórbidos adicionales, resultados de laboratorio y gabinete, grado de inflamación, uso de antibiótico previo, uso de tocolíticos, morbilidad y mortalidad de madre y producto y resultado histológico, con el fin de encontrar el perfil de presentación y poder mejorar la exactitud diagnóstica.

Los casos estudiados presentaron una edad promedio de 29 años, una edad gestacional promedio de 20 semanas (II trimestre), con antecedente de gestas previas de 2.1.

La clínica de los pacientes fue de dolor $100 \%$ el cual se distribuyó de la forma siguiente: un $15 \%$ en flanco derecho,

Revista electrónica publicada por el Departamento de Farmacología de la Escuela de Medicina de la Universidad de Costa Rica, 2060 San José, Costa Rica. ${ }^{\circledR}$ All rights reserved. 
$6.7 \%$ en hipogastrio, 6.7 en hipocondrio derecho y el resto $71.6 \%$ en fosa ilíaca derecha. El siguiente síntoma fue vómito $70 \%$, fiebre $29 \%$, náusea $18 \%$. Al examinar el paciente se encontró $100 \%$ dolor: $15 \%$ en flanco derecho, $5 \%$ en hipocondrio derecho y $10 \%$ en hipogastrio, el resto $70 \%$ en fosa ilíaca derecha. Además, el $70 \%$ de pacientes presentaron rebote, $37 \%$ resistencia en la pared a la palpación y un 14.8 distensión abdominal.

El tiempo promedio de evolución hasta su operación fue de 2.1 días. .

El leucograma promedio fue de 17.113 con un $81 \%$ de formas segmentadas, 6.3 formas en banda. La medición de proteína $C$. reactiva se tomó en 4 casos, pero fue positiva en todos $(100 \%)$ con un promedio de 3.2 , para un normal de $0.5 \mathrm{mg} / \mathrm{dl}$.

El examen general de orina se practicó en $48 \%$, obteniéndose un examen normal en $38 \%$ y en $62 \%$ de los casos mostró conteo de más de 10 leucocitos por campo.

Se realizó ultra sonido en $44 \%$ de las pacientes, fue positivo en $16.6 \%$ y negativo en $83.3 \%$. En ningún caso se obtuvo ultra sonido transvaginal, radiografías convencionales 0 contrastadas, ni tampoco tomografía axial computarizada o resonancia. Las pacientes se abordaron con una incisión

\section{Discusión}

$\mathrm{Si}$ el diagnóstico temprano en toda patología de emergencia es importante, lo es más aún cuando se trata de la mujer embarazada por el riesgo que tiene la pérdida del producto.

Lo esperable va a ser una mujer en los 20s (29 años en este estudio), con un promedio de 2.1 días de evolución de acuerdo a esta revisión, quién consulta paramediana $66.6 \%$, mediana $25 \%$ y transversa en $8.3 \%$.

El grado de inflamación descrito por los cirujanos fue: G-I 18.5\%, G-II 40.7\%, G-III $11.1 \%$, y G-IV 29.6\%. Cuatro de estos pacientes recibieron antibiótico y/o analgésico previamente, en dos de ellos se encontró inflamación G.-III y en los otros dos G-IV.

En el $85 \%$ de los casos se usaron antibióticos y de éstos cefalosporina de 1 er generación en un $56.5 \%$, esquemas combinados con aminoglicósido, antianaerobio y cefalosporinas de 3era en $43.5 \%$.

Se reportó un $14.8 \%$ de apéndice retrocecal. En ningún caso de dejaron drenajes.

En un $44 \%$ de pacientes se usaron tocolíticos, en pacientes con edad gestacional alrededor de 21.5 semanas, En 3 casos los cirujanos tuvieron que proceder con cesárea al mismo tiempo que la apendicectomía..

El reporte de patología fue de: $66.6 \%$ apendicitis aguda simple, $14.8 \%$ apendicitis perforada y $18.5 \%$ apéndices negativas.

La población tuvo una estancia promedio de 5.4 días

No hubo mortalidad del feto ni la madre. La morbilidad fue de $3.7 \%$, una sepsis de herida.

por dolor en el $100 \%$ de los casos, dolor que se diferencia de los dolores uterinos y que con el paso de las horas se caracteriza más y se localiza en cuadrantes derechos dependiendo de la edad gestacional, hasta llegar a flanco o hipocondrio derecho en algunos casos de embarazos muy avanzados (15\% y $6.7 \%$ respectivamente en esta serie). Al

Revista electrónica publicada por el Departamento de Farmacología de la Escuela de Medicina de la Universidad de Costa Rica, 2060 San José, Costa Rica. ® All rights 
examen físico el $100 \%$ de las pacientes presentan dolor, pero hasta un $30 \%$ en localizaciones diferentes a fosa ilíaca derecha.

Si el uso de antibióticos o analgésicos previos al diagnóstico enmascaran, en el caso de pacientes embarazadas es mayor, así vemos como de 4 pacientes que recibieron $t x$, las cuatro estaban ya avanzadas.

Lo esperable es un leucograma elevado (promedio 17.113) pues la paciente embarazada como se ha comentado cursa ya de por sí con cierta leucocitosis, sin embargo ésta no tiene desviación izquierda que si es típico en apendicitis, con bandemia (6.3 en esta serie). La proteína $C$. Reactiva aunque no específica, si es muy sensible en advertirnos de la presencia de un proceso inflamatorio En nuestro caso fue positiva $100 \%$ con un valor muy por encima del normal. Como se comenta en la literatura, el examen general de orina va a presentar en la embarazada una leucocitoria mayor como en nuestro estudio en un $62 \%$ y debe tomarse con cuidado para no tratar el paciente como infección del tracto urinario.

El uso del ultra sonido general fue de muy poca utilidad en nuestro medio, comparado con reportes de la literatura y no se realizaron otros estudios como TAC, gammagrafa, resonancia ,radiografías con protección, etc.

Una vez establecido el diagnóstico, se debe proceder rápidamente con reposo de tubo digestivo, hidratación, analgésicos, antibióticos no tóxicos para el producto (profilácticos en casos G-I y II y terapéuticos en los casos G-III y IV) [5]. No se aconseja el uso de tocolíticos profilácticos de rutina y más bien se debe realizar un control pos-operatorio cuidadoso hasta por 7 días y administrárselos a la paciente que desarrolla actividad uterina [23]. Nosotros usamos antibióticos en el $85 \%$ de los casos, en su mayoría una cefalosporina de 1era generación y solo se presentó una sepsis de herida, a pesar de que el grado de inflamación apreciado por los cirujanos fue de un GIII y IV en un $40 \%$.

$\mathrm{Ya}$ en la sala de operaciones se recomienda una posición de decúbito dorsal con rotación de unos $30^{\circ}$ a la izquierda para evitar la compresión del producto sobre la vena cava, especialmente en el 3er trimestre. La incisión recomendada es la transversa ( $8.3 \%$ ) en este grupo, fue seguida de la paramediana (66.6\% en esta serie) y un $25 \%$ en línea media [6,32]. Evitando mucha manipulación uterina [33]. Sin embargo, si hay peritonitis se debe realizar un lavado exhaustivo dado que no se puede correr el riesgo de un absceso residual. En cualquier caso se deben evitar los drenajes (como se procedió en nuestra casuística), pues estos son cuerpos extraños que producen irritación y pueden estimular la actividad contráctil de la matriz. No se aconseja el uso de uteroinhibidores de rutina $y$ solo cuando la paciente desarrolla alguna actividad contráctil como sucedió en el $44 \%$ de nuestras pacientes.

Si nos enfrentamos a un caso con sepsis franca y embarazo viable, debemos valorar bien la actividad uterina. De manera que si la labor está avanzada se puede inducir el parto e inmediatamente después practicar la laparotomía. En ese mismo caso si la labor es temprana y va a tardar más de 12 horas se puede proceder con la laparotomía y luego con el parto vaginal con anestesia. Pero si estamos ante un caso de choque séptico, donde el

Revista electrónica publicada por el Departamento de Farmacología de la Escuela de Medicina de la Universidad de Costa Rica, 2060 San José, Costa Rica. ® All rights 
producto se considera que estará mejor en un ambiente artificial que en el útero gestante, se debe proceder con cesárea de inmediato y apendicectomía, como se procedió en tres de nuestras pacientes, con limpieza del abdomen al mismo tiempo; dejando inclusive en algunos casos el abdomen abierto para subsecuentes lavados hasta que se considere limpio [5].

\section{Conclusiones}

De lo antes expuesto concluimos que el embarazo induce cambios, que alteran el diagnóstico de ApAg en la paciente embarazada, por lo que debe sospecharse, y cuando se piensa se debe resolver rápido (menos de 24 horas), para evitar una perforación. La evaluación clínica y los exámenes de laboratorio y gabinete se deben elegir los más ágiles y que no retrasen el diagnóstico. El médico debe referir

\section{Bibliografía}

1-Tarranza H.M., Moore R.D: Gynecologic causes of acute abdomen and the acute abdomen in pregnancy. Surg. Clin.North Am 77 (6):1371, 1997.

2-Arclay L. Nighinem HT. Appendectomy during pregnancy increases the risk for adverse fetal outcomes. Am. Coll Surg. 2007; 205: 530-542.

3-babler E.A. Perforative appendicitis complicating pregnancy. JAMA 51:1310,1908.

4-Mazze R.I., Kallen B: Appendectomy during pregnancy. A Swedish Registry Study of 778 cases. Obst Gynecol 77:836, 1991.

5-Sharp H.T., Gastrointestinal Surgical Conditions during pregnancy. Clin.Obst Gynecol 37(2): 1994.
En el caso que se use la laparoscopía, se aconseja usar presiones de neumoperitoneo bajas de $10-12 \mathrm{mmHg}$, entrada con visión directa, control de doppler transvaginal y preferiblemente en el II trimetre. En el tercer trimestre su uso para algunos todavía es controversial. Se aconseja en todos los casos hacerlo con capnografía para evitar problemas de retención de $\mathrm{CO}_{2}$ dañinas para el producto $[11,32]$.

rápidamente estas pacientes para que el cirujano actúe y resuelva oportunamente. Como es de esperarse la estancia hospitalaria en estos casos es francamente mayor de 5.4 días vs. 1.5 días en pacientes no embarazadas, lo que genera un costo mayor en la atención, de modo que entre más temprano se diagnostique y opere, más rápido sale la paciente y a menor costo.

6-Mulhim A.L. Acute Appendicitis in Pregnancy Int.Surg. 81:295-293,1996.

7-Sharp H.T. Gastrointestinal surgical conditions during pregnancy. Clin Obstet Gynecol $37: 306,1994$.

8-Babaknia A, Parsa H, Woodruff J.D. Appendicitis during pregnancy. Obstet Gynecol 50: 40-47, 1977.

9-Gómez A, Wood M. Acute appendicitis during pregnancy. Am J.Surg. 137: 180-183,1979.

10-Horowitz M.D., Goméz G.A.. Aute appendicitis during pregnancy. Diagnosis and management. Arch. Surg. 120:1362-1367,1985.

11-Molpus B.K., Rothrock S.G. Apendicitis aguda, un enfoque práctico para mejorar la

Revista electrónica publicada por el Departamento de Farmacología de la Escuela de Medicina de la Universidad de Costa Rica, 2060 San José, Costa Rica. (®) All rights reserved. 
evolución del paciente. Residents and Staff phicisians 41 (11): 11,1995.

12-Mahmoodian S: Appendicitis complicating pregnancy. South Med.J. 854:19,1992.

13-Fallon W.F., Newman JS,Fallen GF. The surgical management of intra-abdominal inflamatory conditions during pregnancy. Surg. Clin North Am. 75:15, 1995.

14-Maasters K, Levine B.A. Diagnosing Apendicitis during pregnancy. Am.J.Surg 148:768,1984.

15-Bongar F, Londers DV, Lewis F. Differential diagnosis of appendicitis and pelvic inflamatory disease. Am.J.Surg. 150:90-96,1985.

16-Blomquist P.G. et al. Mortality after appendectomy in Sweden. Ann.of Surg.288 (4):455, 2001.

17-Sarason E.L., Bauman S. Acute appendicitis in pregnancy. Difficulty in diagnosis. Obstet Gynecol 22:282, 1963.

18-Hale D.A. Appendectomy. Ann of Surg. 225 (2): 252,1997.

19-Zaldívar FR, Ramírez D, Vásquez J. Propuesta de una escala práctica para el diagnóstico temprano de apendicitis aguda. Cirujano Gnl Vol 19 (4): 274-279, 1997.

20-Boer JL, Reis RA, Arren RA: Appendicitis in pregnancy with changes in position and axis of the normal appendix in pregnancy. JAMA 98: $1359,1932$.

21-Maurad J,Elliot JP, Appendicitis in pregnancy. New information that contradicts lonheld cllinical beliefs. Am J Obstet Gynecol 182 (5):1027-1029,2000.

22-Parungo C.P., Brooks D.C. Care in special situations. The pregnant surgical patient. Am Coll Surg. On line. 2000.

23-Kart B. Ktz WL, Watson WJ. Effect of nonobstetrical operation during pregnancy. Surg. Gynecol Obstet 177:371,1993.

24-Lim. H.K., Bae. S. H., Seo G.S. Diagnosis of acute appendictis in pregnant women. Value of sonography. ARJ Am J. Roentogenol 1992; 159 (3): 5329-42.

25-Castro MA, Shipp T, Castro E. The use of helical computed tomography in pregnancy for the diagnosis of acute appendicitis. Am. J.Obstet Gynecol 184 (5): 954-957,2001.

26-McColl I. More presicion in diagnosing Appendicitis. The New Engl J. Of Med 338 (3):190-191, 1998.

27-Brown M.A., Rirchard K.B., Semelka. Magnetic Resonance evaluation of pregnant patient with acute abdominal pain. Semin Ultrasound CT MR, 2005; 26: 206-211.

28-Shellock F.G., Kanal E. Policies, guidelines and recomendations for M.R. imaging safety and patient managment. SMRI safety committee. J. Magn Reson Imaging, 1991; 1: 97-101.

29-Schriber JH: Laparoscopy appendectomy in pregnancy. Surg. Endosc 4: 100,1990.

30-Spirtos NM Eisenkop TW. Laparoscopy: A diagnostic aid in cases of suspected appendicitis. Am.J. Ostset Gynecol 156: 90, 1987.

31-Wolfe G, Oto A. M.R. Evaluation of acute abdominal pain in pregnant paatients. Appl Radiol 2007; 36 (9): 20-25.

32-Affleek D.G, Handrahan D.L. The laparoscopic management of appendicitis and cholelithiasis during pregnancy. Am.J.Surg. 178: 523-529,1999.

33-Tamir I L, Bongerd FI. Acute appendicitis in the pregnant patient. Am.J.Surg. 160:571,1990. 34-Pastore P.A., Loomis D.M., Surret J. Appendicitdis in pregnancy. J. Am Board Fam Med 2006; 19 (6): 621-626.

35-Hunt MG, Martin JN, Martin RW. Perinatal aspects of abdominal surgery for non obstetric disease.. Am J. Perinatol 6:412,1989.

36-Kammerer WD. Non-ostetric surgery in pregnancy. Med Clin North Am 71:551,1987.

Revista electrónica publicada por el Departamento de Farmacología de la Escuela de Medicina de la Universidad de Costa Rica, 2060 San José, Costa Rica. (®) All rights

reserved. 


\section{Correspondencia:}

\section{Mario Sánchez Arias}

Correo electrónico: masanchezarias@gmail.com

Teléfono: (506) 2812436 Fax 2836171

Revista electrónica publicada por el Departamento de Farmacología de la Escuela de Medicina de la Universidad de Costa Rica, 2060 San José, Costa Rica. ${ }^{\circledR}$ All rights

reserved. 